\title{
Relationships between prolonged neck/shoulder pain and sitting spinal posture in male and female adolescents
}

\section{Introduction}

Neck/shoulder pain (NSP), pain felt in the cervical and upper trapezius region (Kuorinka et al 1987), is a common problem in adults (Croft et al 2001; Palmer et al 2001; Hill et al 2004), incurring high societal and individual costs (Ferrari \& Russell 2003). A less commonly recognised problem is adolescent NSP.

Several studies have estimated the prevalence of adolescent NSP (Wedderkopp et al 2001; Ehrmann Feldman et al 2002; Hakala et al 2002; van Gent et al 2003; Murphy et al 2004; Siivola et al 2004). However, only two studies have estimated the prevalence of recurrent NSP in adolescents (El-Metwally et al 2004; Siivola et al 2004) and none have estimated the prevalence of prolonged NSP, which are both aspects of chronicity. Siivola et al. (2004) noted that $17 \%$ of 16 year olds had experienced recurrent NSP at least once a week during the previous 6 months. Similarly, El-Metwally et al. (2004) reported that $22 \%$ of 12 year olds had experienced NSP both at baseline and one year later. However this measure did not account for disorders lasting less than a year, and ignores the possibility that the pains could be unrelated.

Although the cause of prolonged NSP in adolescents has not been investigated, it is likely to be multi-factorial, including individual physical and psychosocial factors which are 
known to be associated with adult prolonged NSP (Makela et al 1991; Webb et al 2003; Ylinen et al 2004). When considering physical factors, there are indications that spinal posture may be associated with adolescent prolonged NSP. In the frontal plane, spinal asymmetry was found to not predict back and neck pain in adults (Dieck et al 1985) though evidence that scoliosis can cause long-term neurological deficits in adults (Morcuende 2003) does suggest a possible link with chronic pain. In the sagittal plane, adult studies have demonstrated that prolonged NSP may be associated with increased neck flexion postures (Ariens et al 2001; Harrison et al 2004) and altered patterns of muscle activity when sitting (Szeto et al 2005). Moreover, two studies have demonstrated a link between non-prolonged NSP and spinal posture in adolescents (Hertzberg 1985; Murphy et al 2004), while altered adolescent posture has been related to computer use (Straker et al 2007) which in turn has been related to NSP (Harris \& Straker 2000). However, to date, there have been no studies investigating the links between prolonged adolescent NSP and spinal posture.

Such a study would need to avoid certain limitations of recent studies in related areas. Previous adult (Ariens et al 2001; Harrison et al 2004; Szeto et al 2005) and adolescent (Hertzberg 1985) work in this area has not investigated lumbar or pelvic postures in the sagittal plane. However, there is evidence that lumbo-pelvic posture influences thoracic posture (O'Sullivan et al 2006) and thoracic muscle activation patterns (O'Sullivan et al 2002; O'Sullivan et al 2006), which may potentially influence the cervical spine. Furthermore, Falla et al. (2007) reported that neutral lumbo-pelvic posture increases cervical spine muscle activation. Some previous work in adults (Harrison et al 2004) and adolescents (Hertzberg 1985) did not considered gender as a confounder, despite the 
influence of gender on both sitting posture and NSP prevalence in adolescents (Siivola et al 2004; Dunk \& Callaghan 2005) and adults (Bot et al 2005). Some previous adult studies have also not measured spinal posture in sitting (Griegel-Morris et al 1992; Harrison et al 2004; McAviney et al 2005). Sitting measurements are particularly relevant for a study of adolescents, as adolescents spend over a quarter of their waking hours sitting (Jago et al 2005) and sitting is a common aggravating factor for prolonged neck pain in adults (Szeto et al 2005).

Therefore the aims of this study were to evaluate the relationships between cervical, thoracic, lumbar and pelvic sagittal sitting postures and adolescent prolonged NSP, with consideration of gender. We restricted our posture measurements to those in the sagittal plane because whilst the optimal sagittal posture is unknown, frontal plane postures are thought to be optimal if straight but abnormal if not.

\section{Materials and Methods}

\section{Subjects}

Data from 1593 adolescents ( 779 females, 814 males) of mean age 14.1 yrs were collected as part of their participation in the "Raine" child health study (http://www.rainestudy.org.au/). This is a long term project on a range of child health and development issues, which started as a pregnancy cohort in which 2,979 women were enrolled at or before the 18th week of gestation from the antenatal clinics at King Edward Memorial Hospital for Women (KEMH) between 1989 and 1991. The children have been followed at birth, $1,2,3,5,8,10$, and now 14 years of age. The criteria for enrolment in 
the Raine study were gestational age between 16 and 20 weeks, sufficient proficiency in English to understand the implications of participation, an expectation to deliver at $\mathrm{KEMH}$, and an intention to remain in Western Australia so that follow-up through childhood would be possible. A comparison of this cohort with the general population of Western Australia utilising the Western Australian Maternal and Child Health Research Database at Telethon Institute for Child Health Research (Kendall 2003) found the sample to be reasonably representative of Western Australians of similar age with the exception of higher 'at risk' pregnancies. There were no exclusion criteria for this section of the cohort. Of the 2868 subjects originally drawn into the study, 1608 attended for the 14 year data collection. Of these, 15 failed to give NSP data for unknown reasons. Data from the 1593 adolescents with full NSP pain and posture data are presented here. The mean (SD) height for these 1593 subjects was $1.64 \mathrm{~m}(0.08)$ and mean weight was $57.6 \mathrm{~kg}$ (13.2).

\section{Procedure}

Children participating in a regular cohort follow-up completed the questionnaire on a laptop at the assessment centre with a research assistant close by to provide assistance if needed. The questionnaire contained 130 questions concerning a broad range of physical, medical, nutritional, psychosocial and developmental issues. The questions relevant to prolonged NSP are recorded below, with the possible responses in parentheses. Subjects who had experienced NSP lasting more than three months at any point in the past and who had also experienced NSP in the past month were classified as having prolonged NSP. 
Did your neck / shoulder pain last for more than 3 months? ("yes" or "no")

Has your neck / shoulder been painful in the last month? ("yes" or "no")

The full child questionnaire took about 1 hour to complete, and the two questions described occurred in the first half.

A physical assessment of the child was carried out after completion of the questionnaire. This measured anthropometric factors, muscle performance, co-ordination and spinal / pelvic posture during sitting, some of which was used for the current study. Spinal sagittal posture was assessed through photographic analysis of visual markers placed on bony landmarks. We have found fair (Fleiss 1986) inter-rater reliability for most of the posture variables used in this study (ICCs $0.4-0.75$ ), except for cervico-thoracic and trunk angle variables, which were good (ICCs 0.75-0.9) (Fleiss 1986). We have also found excellent (Fleiss 1986) re-digitisation reliability (ICCs $>0.9$ ) (Perry et al in press) which, together with standard errors of measurement (SEMs) of $3.2^{\circ}$ to $6.5^{\circ}$, support its use for large cohort studies.

Retro-reflective markers were placed on the right outer canthus, right tragus, C7 and T12 spinous processes, anterior superior iliac spine and greater trochanter. Lateral photographs were taken with each child sitting on a stool (adjusted to their popliteal height) during three different static sitting postures: a) looking straight ahead, b) looking down at their lap, and c) sitting slumped. To minimise parallax error, the camera (Olympus FE-130, Tokyo, Japan) was positioned on a tripod $80 \mathrm{~cm}$ from the floor and $250 \mathrm{~cm}$ from the subject, with the subject aligned so they were facing perpendicular to 
camera. Four trained health professionals collected the posture images (image resolution 2594 x 1944 pixels). Marker points were digitised using a Peak Motus motion analysis system (Version 8; Peak Performance Technologies, Inc., Centennial, CO, USA) and standard head/neck/thoracic angles were calculated, as defined in Figure 1. Identical or similar angles have been used in other studies (O'Sullivan 2006; Straker et al 2008).

\section{INSERT FIGURE 1 ABOUT HERE}

\section{Data analysis}

Statistical analysis was carried out with SPSS version 13 (SPSS Inc., Chicago, USA). The prevalence of prolonged NSP was calculated as a percentage of the whole cohort. Descriptive statistics were performed on the prolonged pain prevalence data. Chi squared analysis was used to assess differences between genders for prevalence values. The difference between usual and slumped sitting was calculated to determine how close adolescents sat to their end of flexion range. Independent t- tests were used to analyse differences in posture variables between pain and no pain groups, and males and females. The effect of individual posture variables on prolonged NSP after controlling for gender differences was assessed using logistic regression models (entry method). Adequate model fit was assessed using the model chi-squared likelihood-ratio test and HosmerLemeshow goodness of fit test. Analysis of residuals was performed to check for cases with undue influence over model estimates. The strength of the predictive ability of the

model was estimated by Nagelkerke $\mathrm{R}^{2}$. Alpha probability level was set at $\mathrm{p}<0.05$ for all comparisons. 


\section{Ethics}

The study was approved by the Human Research Ethics Committee of Curtin University of Technology and Princess Margaret Hospital, Perth.

\section{Results}

\section{Prevalence}

85 subjects $(5.3 \%$ of the sample of 1593 with complete NSP data) reported both recent NSP and at least one episode of NSP that had lasted three or more months. Prolonged NSP was reported by 51 females $(6.5 \%$ of 779$)$ and by 34 males ( $4.2 \%$ of 814$)$. The gender difference was significant $(p=0.035)$.

\section{Posture}

Gender differences in habitual sitting spinal posture were detected, with females in more erect and lordotic postures than males when looking straight ahead (Table 1). The statistically significant differences between genders were in most cases of moderate or large effect size. For example, the $10.2^{\circ}$ difference in lumbar angle between genders represented 0.58 of the averaged standard deviation of the two groups, which is interpreted as a small/moderate gender effect, while the $12.7^{\circ}$ difference in trunk angle between genders represented 1.14 of the averaged standard deviation of the two groups, which is interpreted as a moderate/large gender effect (Hopkins 2007). 
INSERT TABLE 1 ABOUT HERE

\section{Prolonged NSP and posture}

Adolescents with prolonged NSP sat with a more flexed cervico-thoracic angle (prolonged NSP mean (SD) of $146.9^{\circ}(8.6)$, no prolonged NSP $149.1^{\circ}(8.3), \mathrm{t}_{\mathrm{df}}=2.19_{1463}$, $\mathrm{p}=0.028$ ), a more extended trunk angle (prolonged NSP $229.4^{\circ}(12.5)$, no prolonged NSP $\left.232.4^{\circ}(12.8), \mathrm{t}_{\mathrm{df}}=1.98_{1463}, \mathrm{p}=0.048\right)$, a more lordotic lumbar angle (prolonged NSP $123.2^{\circ}(18.6)$, no prolonged NSP $\left.129.6^{\circ}(18.4), \mathrm{t}_{\mathrm{df}}=2.91_{1466}, \mathrm{p}=0.004\right)$, and more anterior pelvic tilt (prolonged NSP 9.7 $(16.4)$, no prolonged NSP $4.3^{\circ}(15.6), \mathrm{t}_{\mathrm{df}}=-2.83_{1466}$, $p=0.005$ ) than those with no experience of prolonged NSP, when looking straight ahead (see Table 2). These differences represented small to moderate effects of protracted NSP on posture measures (Cohen's-d 0.26, 0.24, 0.35 and 0.33 respectively (Hopkins 2007)).

There was no statistically significant difference in head flexion $(\mathrm{p}=0.689)$, neck flexion $(p=0.555)$ or craniocervical angle $(p=0.915)$ between adolescents who had and had not experienced prolonged NSP, when sitting looking straight ahead (see Table 2).

\section{INSERT TABLE 2 ABOUT HERE}

\section{INSERT TABLE 3 ABOUT HERE}

\section{INSERT TABLE 4 ABOUT HERE}

A similar pattern of postures were observed across the two groups when subjects were sitting looking down, though this time the differences between cervico-thoracic angle and 
trunk angle were not significant (Table 3). There were no significant pain differences for the variable representing how close to the end of range adolescents sat (difference between usual and slump sitting) (Table 4) as there were differences in slump posture similar to usual posture (data not shown).

\section{Prolonged NSP, posture and gender}

As there were moderate to large gender differences in posture variables, logistic regression was used to examine the predictive value of each posture variable in which there was a statistically significant difference between those subjects with and without prolonged NSP, controlling for gender.

When subjects were looking straight ahead, both increased anterior pelvic tilt $(\mathrm{p}=0.019$, Odds Ratio $(\operatorname{Exp}(\beta))=1.02,95 \% \mathrm{CI}$ : 1.00-1.03, Nagelkerke's $\left.\mathrm{R}^{2}=0.021\right)$ and decreased lumbar angles (increased lordosis) $(\mathrm{p}=0.014$, Odds Ratio $(\operatorname{Exp}(\beta))=0.99,95 \% \mathrm{CI}$ : 0.97-1.00, Nagelkerke's $\mathrm{R}^{2}=0.017$ ) were weakly predictive of prolonged NSP after controlling for gender, whilst in contrast to the unadjusted analyses, trunk angle ( $p=$ 0.290, Odds Ratio $(\operatorname{Exp}(\beta))=0.99,95 \% \mathrm{CI}$ : 0.97-1.01) and cervico-thoracic angle $(\mathrm{p}=$ 0.156, Odds Ratio $(\operatorname{Exp}(\beta))=0.98,95 \% \mathrm{CI}: 0.95-1.01)$ were not. When subjects were looking down, both increased anterior pelvic tilt $(\mathrm{p}=0.020$, Odds Ratio $(\operatorname{Exp}(\beta))=1.02$, 95\%CI: $1.00-1.04)$ and decreased lumbar angle $(\mathrm{p}=0.025$, Odds Ratio $(\operatorname{Exp}(\beta))=0.99$, 95\%CI: 0.97-1.00) were again weakly predictive of prolonged NSP after controlling for gender, whilst trunk angle and cervico-thoracic angle were not $(\mathrm{p}=0.332$, Odds Ratio $(\operatorname{Exp}(\beta))=0.99,95 \% \mathrm{CI}: 0.97-1.01)$, and $\mathrm{p}=0.396$, Odds Ratio $(\operatorname{Exp}(\beta))=0.99,95 \% \mathrm{CI}$ :

0.96-1.02), respectively). Finally, after adjustment for gender, there were no significant 
associations between prolonged pain and any of the variables representing how close to the end of range adolescents sat (difference between usual and slump sitting). Due to rater effects being found in posture assessment reliability, models including rater were also tested, but did not alter the relationship between pelvic and lumbar posture and prolonged neck and shoulder pain.

There were no significant interaction effects between gender and any posture variable on prolonged NSP. Figure 2 illustrates the relationship between prolonged NSP, posture variables and gender.

\section{INSERT FIGURE 2 ABOUT HERE}

\section{Discussion}

\section{Prevalence of prolonged NSP in adolescents}

Although previous work has suggested that adolescent NSP may be recurrent (ElMetwally et al 2004; Siivola et al 2004) the present study is the first to show that it can also be prolonged, lasting over 3 months in $8 \%$ of the sample. These findings indicate that almost one in ten adolescents report that they are experiencing periods of prolonged NSP, which is of concern. Indeed, this figure may be an underestimation. Some subjects

may have interpreted brief intervals without NSP as the end of a continuous episode, and so may not have reported any episodes of long-term pain punctuated by short pain-free periods. We also repeated the analysis described in this paper with just NSP of greater than three months duration regardless of whether they experienced NSP in the last month 
and found very similar results. In a follow up of these adolescents at 17 years of age we are asking separate questions concerning 3 months of continuous pain and 3 months of intermittent pain.

Prolonged NSP was more common in females than in males. This gender difference is consistent with the higher prevalence of non-prolonged NSP in female adult (Bot et al 2005) and adolescent populations (Siivola et al 2004) and indicates that this pattern is evident at early adolescence.

\section{Habitual sitting postures and pain}

Clear gender differences existed in usual adolescent sitting posture, with females sitting in more erect spinal postures than males, which echoes previous findings in adults (O'Sullivan et al 2006). When males and females were analysed together, those with prolonged NSP had similar postural patterns to the whole female group. Since females had a greater prevalence of prolonged NSP, this suggested that some of the differences in postures between pain and non-pain groups could be explained simply by gender. This was supported by fewer significant differences between pain and pain free groups when the genders were split (Table 2), and confirmed by the logistic regression analysis controlling for gender. This demonstrated that with gender controlled, only an increase in lumbar extension angle and increased anterior pelvic tilt in sitting were associated with prolonged NSP in these subjects. This was the case both when looking straight ahead and when looking down, indicating that these findings may be applicable to a range of sitting positions. These are the first reports of such associations in adolescents and highlight the importance of controlling for gender when analysing for associations between posture 
and pain. Interestingly the adolescents with pain did not sit closer to their end of flexion range as has been found with adults with low back pain (O'Sullivan 2006)

Males and females appeared to have a similar qualitative relationship between posture and prolonged NSP. This was supported by no significant gender/posture interaction effects and can be seen in Figure 2. Whether the underlying mechanisms contributing to NSP are the same across genders is, however, unknown.

The findings of this study do not support the clinical belief that prolonged NSP in adolescents is associated with altered cervico-thoracic postures. This is in contrast to previous adult findings that prolonged neck pain disorders are associated with protracted and flexed head/neck postures (Harrison et al 2004). Two other studies (Ariens et al 2001; Szeto et al 2005) also showed a non-significant trend for such an effect. As differences in cervico-thoracic posture were not observed in the current study population once gender was controlled for, it is possible that gender may have been a confounding factor in the study by Harrison and colleagues (2004), where gender corrections were not performed. However, Ariens et al (2001) did adjust for gender and Szeto et al (2005) used only female subjects, so other factors may be important. We do not believe that our differing results are due to inferior reliability or sensitivity of posture measures, as our reliability was fair to good, and our sample size was large enough to detect small differences. Pain definitions in our study were also similar to those in the other two studies. A more likely reason for the differences in findings was the adolescent age group in our study. Adolescents and adults may have different musculoskeletal tissue characteristics (Lambertz 2003), so it seems reasonable that they might respond 
differently to similar postures. It is also possible that sub-groups with differing cervicothoracic postures exist in the prolonged NSP group in our study, which were lost in the current whole-group analysis due to a wash-out effect (where effects in different directions for sub-groups add together to give the impression of no effect for the combined groups). Such an effect has been reported previously in studies investigating spinal posture and prolonged low back pain (Dankaerts et al 2006). Our results also differ from adolescent NSP studies not specifically investigating prolonged NSP (Hertzberg 1985; Murphy et al 2004), which may result from different mechanisms for prolonged pain. Different posture assessment methods may also contribute to the different findings.

This study identified greater lumbar lordosis and the associated anterior pelvic tilt as the only postural difference between those with prolonged NSP and those without. These findings likely reflect that the adolescents with prolonged NSP tended to have more lordotic spines, such that they sat with more lordosis and their slump position was more lordotic than pain free subjects. The fact that there was no difference between usual and slump sitting between groups would suggest that this is a structural difference rather than a motor response to pain, further supported by the fact that there was no report of pain during the posture measure collection. No previous studies have investigated lumbopelvic posture in relation to prolonged NSP, although an increased lumbar lordosis has been associated with a prolonged low back pain subgroup (Dankaerts et al 2006). It is not clear how this increased lordosis influences NSP although a recent study demonstrated that changes in lumbo-pelvic posture significantly alter motor control patterns in the neck in pain free subjects (Falla et al 2007). It is possible that the increased lumbar lordosis in 
the prolonged NSP subjects observed in this study influences motor control of the neck in a similar manner. However it is not known whether these changes in lumbo-pelvic posture contribute to NSP or are a result of NSP in these subjects. We are currently conducting longitudinal work aimed at clarifying causality and mechanism issues. We are also investigating the relationship between posture and low back pain, and the comorbidity of NSP and low back pain.

The Nagelkerke's $R^{2}$ values of the logistic regression models was very small. The lack of association between NSP and cervico-thoracic posture and the weak association with lumbo-pelvic posture, indicate that posture may have only a small influence on prolonged NSP. Indeed, gender appears to have a greater association with posture than pain. It also suggests that there may be other risk factors of greater importance, or that sub-groups exist where postural factors are important, which is a focus of our ongoing research. These findings initially suggest limited support for the application of generic postural education for the management of prolonged NSP in adolescents. However, although the magnitude of the posture changes was very small, it is possible that even small systematic differences in habitual posture early in life may lead to a large increase in accumulated load. Thus the small differences identified in the study, which may not be considered clinically important at one point of time, may have long term health consequences. Hence further work is necessary before the efficacy of postural education can be challenged.

In common with other literature, one limitation of this work is that prolonged NSP has been used as a single outcome variable despite the fact that it may have several different presentations and levels of impairment. No indication as to the intensity of pain or 
impairment was made from this study although this will be a focus of our longitudinal research on this cohort, with questions assessing the impact of pain on function, school/work attendance, and the need to take medication or seek professional advice. Another possible limitation is that although our measures showed good sensitivity in terms of being able to detect very small group differences, they may have been limited by the biological variability (extent to which test postures approximated habitual posture), the validity (in comparison to other imaging technologies) and the precision limits of the photographic method. Another limitation is the whole-group analysis. Different subgroups of NSP may exist that have different anatomical, physiological or psychological mechanisms, and therefore different risk factors that underlie the aetiology of the pain disorder. It is also possible that altered spinal posture interacts differently with these variables. For example, cervico-thoracic posture may only be an important risk factor for a sub-group with high exposure to computers and poor motor control. Future work will therefore aim to sub-classify prolonged NSP and examine multivariate relationships within each sub-group.

\section{Conclusion}

Prolonged NSP affected $8 \%$ of adolescents, and was more common in females than males. Prolonged NSP was weakly associated with more lordotic lumbo-pelvic postures, but the clinical belief that NSP is related to cervico-thoracic postures was not supported when gender was included in the model. 


\section{Acknowledgements}

We would like to acknowledge funding from the Australian National Health and Medical Research Council (project \# 323200), the Raine Foundation at the University of Western Australia, Healthway, the Arthritis Foundation of Australia, and the Arthritis Foundation of Western Australia. We would also like to thank Rosemary Austin, Lee Clohessy, Alex D'Vauz, Clare Haselgrove, Monique Robinson, Nick Sloan and Diane Wood for collection and/or initial processing of data. 


\section{Legends for Figures:}

Figure 1. Illustrations of Postural Angles. Arrows indicate the angle measured. Dashed lines indicate the vertical. Angle definitions were as follows. Head flexion was the angle formed between the vertical and the line from canthus to tragus (measured above intersect); neck flexion was the angle formed between the vertical and the line of tragus to C7 (measured above intersect); craniocervical angle was formed between the lines of canthus to tragus and tragus to $\mathrm{C} 7$ (measured anterior to intersect); Cervico-thoracic angle was formed between the lines of tragus to C7 and C7 to T12 (measured anterior to intersect); thoracic flexion was formed between the vertical and the line of C7 to T12 (measured from vertical above intersect); trunk angle was formed between the lines of C7 to T12 and T12 to greater trochanter (measured posterior to intersect); lumbar angle was formed between the lines of T12 to ASIS and ASIS to greater trochanter (posterior angle); and pelvic angle was formed between the vertical and the line of greater trochanter to ASIS (measured from vertical above intersect).

Figure 2. Boxplots displaying the differences between prolonged NSP and no prolonged NSP groups (degrees) in males and females for gravity-referenced posture variables. 


\section{References}

Ariens G, Bongers P, Douwes M, Miedema M, Hoogendoorn W, van der Wal G, Bouter L, van Mechelen W. Are neck flexion, neck rotation, and sitting at work risk factors for neck pain? Results of a prospective cohort study. Occupational and Environmental Medicine 2001; 58(3): 200-207

Bot S, van der Waal J, Terwee C, van der Windt D, Schellevis F, Bouter L, Dekker J. Incidence and prevalence of complaints of the neck and upper extremity in general practice. Annals of the Rheumatic Diseases 2005; 64: 118-123

Croft PR, Lewis M, Papageorgiou AC, Thomas E, Jayson MI, Macfarlane GJ, Silman AJ. Risk factors for neck pain: a longitudinal study in the general population. Pain 2001; 93(3): 317-325

Dankaerts W, O'Sullivan PB, Burnett AF, Straker LM. Differences in sitting posture are associated with non-specific chronic low back pain disorders when patients are sub-classified. Spine 2006; 31(6): 698-704

Dieck GGS, Kelsey JJL, Goel VVK, Panjabi MMM, Walter SSD, Laprade MMH. An epidemiologic study of the relationship between postural asymmetry in the teen years and subsequent back and neck pain. Spine 1985; 10(10): 872-877

Dunk NM, Callaghan JP. Gender-based differences in postural responses to seated exposures. Clinical Biomechanics 2005; 20: 1101-1110 
Ehrmann Feldman D, Shrier I, Rossignol M, Abenhaim L. Risk factors for the development of neck and upper limb pain in adolescents. Spine 2002; 27(5): 2328

El-Metwally A, Salminen JJ, Auvinen A, Kautiainen H, Mikkelsson M. Prognosis of non-specific musculoskeletal pain in preadolescents: A prospective 4-year followup study till adolescence. Pain 2004; 110(3): 550-559

Falla D, O'Leary S, Fagan A, Jull G. Recruitment of the deep cervical flexor muscles during a postural-correction exercise performed in sitting. Manual Therapy 2007; 12: $139-143$

Ferrari R, Russell AS. Neck pain. Best Practice \& Research. Clinical Rheumatology $2003 ; 17: 57-70$

Fleiss JL. The design and analysis of clinical experiments, 1. New York: John Wiley, 1986; ch 1, p7

Griegel-Morris P, Larson K, Mueller-Klaus K, Oatis CA. Incidence of common postural abnormalities in the cervical, shoulder, and thoracic regions and their association with pain in two age groups of healthy subjects. Physical Therapy 1992; 72(6): $425-431$

Hakala P, Rimpela A, Salminen JJ, Virtanen SM, Rimpela M. Back, neck, and shoulder pain in Finnish adolescents: national cross sectional surveys. British Medical Journal 2002; 325: 743-747

Harris C, Straker L. Survey of physical ergonomics issues associated with school children's use of laptop computers. International Journal of Industrial Ergonomics 2000; 26(3): 337-346 
Harrison DD, Harrison DE, Janik TJ, Cailliet R, Ferrantelli JR, Haas JW, Holland B. Modeling of the sagittal cervical spine as a method to discriminate hypolordosis: results of elliptical and circular modeling in 72 asymptomatic subjects, 52 acute neck pain subjects, and 70 chronic neck pain subjects. Spine 2004; 29(22): 24852492

Hertzberg A. Prediction of cervical and low-back pain based on routine school health examinations. A nine- to twelve-year follow-up study. Scandinavian Journal of Primary Health Care 1985; 3(4): 247-253

Hill J, Lewis M, Papageorgiou AC, Dziedzic K, Croft P. Predicting persistent neck pain: A 1-year follow-up of a population cohort. Spine 2004; 29(15): 1648-1654

Hopkins WG. 2007 A new view of statistics. From http://sportsci.org/resource/stats/index.html (last accessed 6/12/07)

Jago R, Anderson CB, Baranowski T, Watson K. Adolescent patterns of physical activity differences by gender, day, and time of day. American Journal of Preventive Medicine 2005; 28(5): 447-452

Kendall GE (2003). Children in families in communities: A modified conceptual framework and an analytic strategy for identifying patterns of factors associated with developmental health problems in childhood. Perth, University of Western Australia. Unpublished PhD thesis.

Kuorinka I, Jonsson B, Kilbom A, Vinterverg H, Biering-Sorensen F, Andersson G, Jorgensen K. Standardised Nordic questionnaires for the analysis of musculoskeletal symptoms. Applied Ergonomics 1987; 18(3): 233-237 
Lambertz D, Mora, I.,Grosset, J.F., Perot,C. Evaluation of musculotendinous stiffness in prepubertal children and adults, taking into account muscle activity. Journal of Applied Physiology 2003; 95(1): 64-72

Makela M, Heliovaara M, Sievers K, Impivaara O, Knekt P, Aromaa A. Prevalence, determinants, and consequences of chronic neck pain in Finland. American Journal of Epidemiology 1991; 134(11): 1356-1367

McAviney J, Schulz D, Bock R, Harrison DE, Holland B. Determining the relationship between cervical lordosis and neck complaints. Journal of Manipulative and Physiological Therapeutics 2005; 28(3): 187-193

Morcuende JA, Dolan, L.A., Vazquez, J.D., Jirasirakul, A., Weinstein, S.L. A prognostic model for the presence of Neurogenic Lesions in atypical idiopathic scoliosis. Spine 2003; 29: 51-58

Murphy S, Buckle P, Stubbs D. Classroom posture and self-reported back and neck pain in schoolchildren. Applied Ergonomics 2004; 35: 113-120

O'Sullivan P, Dankaerts W, Burnett A, Straker L, Bargon G, Moloney N, Perry M, Tsang S. Lumbopelvic kinematics and trunk muscle activity during sitting on stable and unstable surfaces. Journal of Orthopaedic and Sports Physical Therapy 2006; 36(1): $19-25$

O'Sullivan P, Dankaerts W, Burnett AF, Farrell GT, Jefford E, Naylor C, O'Sullivan KJ. Effect of different upright sitting postures on spinal pelvic curvature and trunk muscle activation in a pain-free population. Spine 2006; 31(19): 707-712 
O'Sullivan PB, Grahamslaw KM, Kendell M, Lapenskie SC, Moller NE, Richards KV. The effect of different standing and sitting postures on trunk muscle activity in a pain-free population. Spine 2002; 27: 1238-1244

O'Sullivan PB, Mitchell, T., Bulich, P. The relationship between posture and back muscle endurance in industrial workers with flexion-related low back pain. Manual Therapy 2006; 11: 264-271

Palmer KT, Walker-Bone K, Griffin MJ, Syddall H, Pannett B, Coggon D, Cooper C. Prevalence and occupational associations of neck pain in the British population. Scandinavian Journal of Work, Environment \& Health 2001; 27(1): 49-56

Perry M, Smith AJ, Straker LM, Coleman JL, O'Sullivan PB. Reliability of sagittal photographic spinal posture assessment in adolescents. Advances in Physiotherapy in press:

Siivola S, Levoska S, Latvala K, Hoskio E, Vanharanta H, Keinanen-Kiukaanniemi S. Predictive factors for neck and shoulder pain: a longitudinal study in young adults. Spine. 2004; 29(15): 1662-1669

Straker L, Burgess-Limerick R, Pollock C, Murray K, Netto K, Coleman J, Skoss R. The impact of computer display height and desk design on 3D posture during information technology work by young adults. Journal of Electromyography and Kinesiology 2008; 18 (2): 336-349

Straker L, O'Sullivan PB, Smith A, Perry MC. Computer use and habitual spinal posture in Australian adolescents. Public Health Reports 2007; 122(5): 634-643 
Szeto GPY, Straker LM, O'Sullivan PB. A comparison of symptomatic and asymptomatic office workers performing monotonous keyboard work--2: Neck and shoulder kinematics. Manual Therapy 2005; 10(4): 281-291

van Gent C, Dols JJ, de Rover CM, Hira Sing RA, de Vet CW. The weight of schoolbags and the occurrence of neck, shoulder, and back pain in young adolescents. Spine 2003; 28(9): 916-921.

Webb R, Brammah T, Lunt M, Urwin M, Allison T, Symmons D. Prevalence and predictors of intense, chronic, and disabling neck and back pain in the UK general population. Spine 2003; 28(11): 1195-1202

Wedderkopp N, Leboeuf-Yde C, Andersen LB, Froberg K, Hansen HS. Back pain reporting pattern in a Danish population-based sample of children and adolescents. Spine 2001; 26(17): 1879-1883

Ylinen J, Salo P, Nykanen M, Kautiainen H, Hakkinen A. Decreased isometric neck strength in women with chronic neck pain and the repeatability of neck strength measurements. Archives of Physical Medicine in Rehabilitation 2004; 85: 1303 1308 